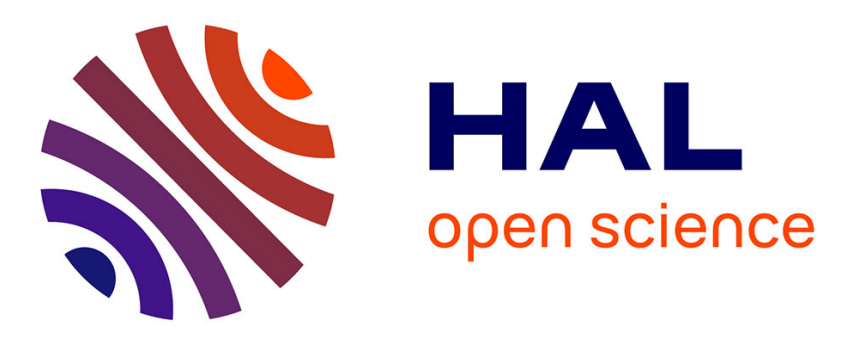

\title{
An experimental performance study of an original ranging protocol based on an IEEE 802.15.4a UWB testbed
}

Réjane Dalce, Adrien van den Bossche, Thierry Val

\section{To cite this version:}

Réjane Dalce, Adrien van den Bossche, Thierry Val. An experimental performance study of an original ranging protocol based on an IEEE 802.15.4a UWB testbed. IEEE International Conference on Ubiquitous Wireless Broadband - ICUWB 2014, Sep 2014, Paris, France. pp. 7-12. hal-01147259

\author{
HAL Id: hal-01147259 \\ https://hal.science/hal-01147259
}

Submitted on 30 Apr 2015

HAL is a multi-disciplinary open access archive for the deposit and dissemination of scientific research documents, whether they are published or not. The documents may come from teaching and research institutions in France or abroad, or from public or private research centers.
L'archive ouverte pluridisciplinaire HAL, est destinée au dépôt et à la diffusion de documents scientifiques de niveau recherche, publiés ou non, émanant des établissements d'enseignement et de recherche français ou étrangers, des laboratoires publics ou privés. 


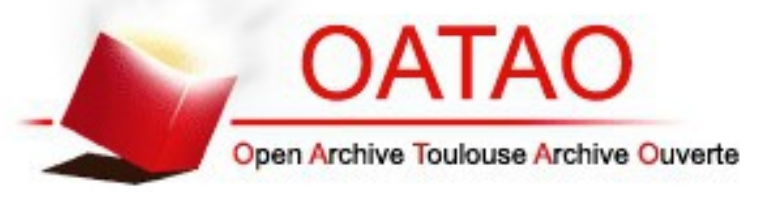

\section{Open Archive TOULOUSE Archive Ouverte (OATAO)}

OATAO is an open access repository that collects the work of Toulouse researchers and makes it freely available over the web where possible.

This is an author-deposited version published in : http://oatao.univ-toulouse.fr/ Eprints ID : 13039

To link to this article : DOI :10.1109/ICUWB.2014.6958942

URL : http://dx.doi.org/10.1109/ICUWB.2014.6958942

To cite this version : Dalce, Rejane and Van den Bossche, Adrien and Val, Thierry An experimental performance study of an original ranging protocol based on an IEEE 802.15.4a UWB testbed. (2014) In: IEEE International Conference on Ubiquitous Wireless Broadband ICUWB 2014, 1 September 2014 - 3 September 2014 (Paris, France).

Any correspondance concerning this service should be sent to the repository administrator: staff-oatao@,listes-diff.inp-toulouse.fr 


\title{
An experimental performance study of an original ranging protocol based on an IEEE 802.15.4a UWB testbed
}

\author{
Rejane Dalce, Adrien van den Bossche, Thierry Val \\ Institut de Recherche en Informatique de Toulouse \\ UMR 5505 - CNRS, Université de Toulouse \\ IUT Blagnac, 1 pl. Georges Brassens BP60073 F-31703 Blagnac Cedex France \\ Email: $\{$ dalce,vandenbo,val $\} @$ irit.fr
}

\begin{abstract}
In this article, we study the behaviour of an UltraWide Band (UWB) physical layer when executing our protocol Parallel Symmetric Double-Sided Two-Way Ranging. We compare it to the reference protocol introduced in IEEE 802.15.4a, Symmetric Double-Sided Two-Way Ranging, in terms of precision of the obtained estimates and to other existing protocols such as SDS-TWR-MA and D-TWR in terms of overhead. These samples were obtained using a testbed made of IEEE 802.15.4a UWB nodes. From these first experiments, we derive a simple correction mechanism which reduces the localisation error compared to the case where no dynamic correction takes place. The location error reduction varies between 41 and $60 \%$ while the algorithm manages to estimate the position $99.9 \%$ of the time with the addition of the correction.
\end{abstract}

\section{INTRODUCTION}

It has been many years since the scientific community has started studying the issue of localisation in the context of the Internet of Things (IoT). Many propositions have targeted Wireless Sensor Networks (WSNs). Although rangefree solutions are usually simple enough to be implemented directly on the sensor nodes, many researchers focus on rangebased methods as they offer better performance. Unfortunately, this performance comes at a certain cost: in order to remove error from real-time measurements, sophisticated algorithms are used which often require a central server. This may yield very good results but this additional cost cannot be overlooked when planning a real-life deployment.

Our objective is to enable fast and cost-effective deployment of indoor WSN-based localisation solutions. We achieve this by integrating our ranging protocol in the existing protocol stack and developing simple algorithms which are suitable for the targeted platforms. In this article, we study the impact of our proposed protocol on the ranging performance of an UltraWide Band (UWB) physical layer. We identify the need for an adjustment of the range measurements by the mobile node, despite the common belief that UWB would be the Graal of localisation.

Following this introduction, we briefly present in section II relevant protocols for TOF-based ranging. Then, we describe the challenge of integrating localisation in a real WSN and our proposed protocol in section III. We show the results obtained using a real UWB testbed in section IV and we finally conclude and present future work in section $\mathrm{V}$.

\section{RELATED WORK}

In 2007, IEEE proposed two PHY layers for low rate, low power WSNs. The first one, CSS, was included as it supported long distance links at high speed. The second one, UWB, was supposed to bring localisation capability to the WSN. As a matter of fact, the research community did not wait for these two technologies to design solutions for wireless network. In [2], signal power and SNR are used to calculate position through a fingerprinting approach. The work described in [3] consists of Time Of Flight estimation using cross-correlation between two nodes modified in order to use the complete IEEE 802.15.4 band. In both [4] and [5], the concept of rings is used to localise mobile nodes. The rings facilitate the computation of the position but they are often built using unreliable sources such as the received signal strength indication (RSSI).

The particularity of UWB is the expected ranging precision. This word refers to the relative proximity of the range estimates to one another while accuracy is used to describe the distance between the estimate and the truth. UWB uses very short pulses to measure the Time Of Flight (TOF). The uncertainty is therefore small which results in precise measurements. But unfortunately, this does not necessarily imply high accuracy. Accuracy is a matter of clock granularity mixed with first path recognition and environmental influences. In order to reduce the influence of clock drift, Symmetric Double-Sided Two-Way Ranging (SDS-TWR) was introduced in [1]. SDS-TWR calls for the execution of Two-Way Ranging twice: the first TWR is led by the mobile and the second by the anchor on figure 1 . One last message is necessary in order to share the timestamps collected by the anchor with the mobile. The existence of a PHY layer suitable for both WSNs and localisation encouraged the study of alternative ranging protocols such as [6],[7] and [8]. These protocols have a common goal which is to improve precision by multiplying the measurements. In Double TWR (D-TWR) [6], the mobile sends the start message twice while the anchor replies once. In [7] (Burst), the mobile sends the first message $k$ times. The anchor also replies $k$ times and finally the mobile acknowledges each message. In [8], the anchor replies $k$ times to the mobile thus the name SDS-TWR Multiple Acknowledgement (SDS-TWR-MA).

Our aim is to design a protocol suite and algorithms which will take advantage of the ranging capability of the PHY layer. Instead of modifying the radio transceiver, we characterize its 


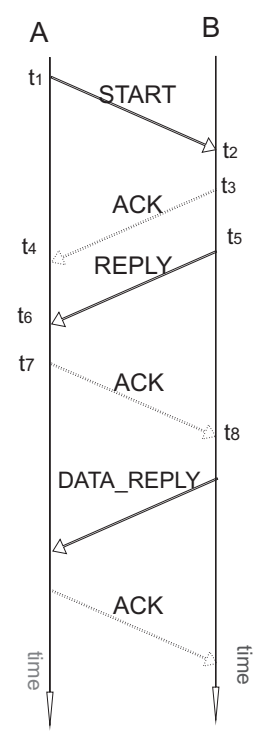

Fig. 1. Symmetric Double-Sided Two-Way Ranging

functions and allow the upper layers to make the best decisions based on this knowledge. We strive to keep this information to a minimal size as our objective is autonomous localisation, that is the mobile node will compute its own position based on real-time measurements. In [9], we proposed a protocol called Parallel Double-Sided Two-Way Ranging (PDS-TWR) that lowers the cost of ranging in terms of overhead and energy consumption. The protocols introduced in [10] share common goals with our solution, specifically, reusing frames to reduce the overhead. Aside from this, the structure is completely different: The network is dedicated to tracking: the anchors periodically broadcast ranging requests during tg. In the Broadcasted Requests and Responses - Immediate ACK (BRR-IA) scheme, each neighbouring mobile node replies in a separate slot and immediately receives the acknowledgements from all the anchors. In the Broadcasted Requests and Responses Broadcasted ACK (BRR-BA) scheme, the acknowledgements are only sent once at the end of the process. In [11], the authors introduce a time structure for the network but all operations are scheduled by the PicoNet Coordinator (PNC). Specifically, the PNC is in charge of allocating Guaranteed Time Slots for localisation between a mobile and its neighbouring anchors. This goes against our approach which is to only involve a limited number of nodes in the localisation process by only relying on local communications to arrange a meeting between the mobile and the anchors.

We designed a ring-based algorithm named inter-Ring Localisation Algorithm (iRingLA) since this approach leads to simpler computations which can be supported by sensor nodes, even the more constrained ones. Once PDS-TWR ended, iRingLA was applied to the collected data and we noticed a need for further data correction. In this paper, we study the behaviour of our ranging tool and propose a strategy to cope with the errors.

We will now introduce our protocols and the preliminary results which will be the building blocks of our solution.

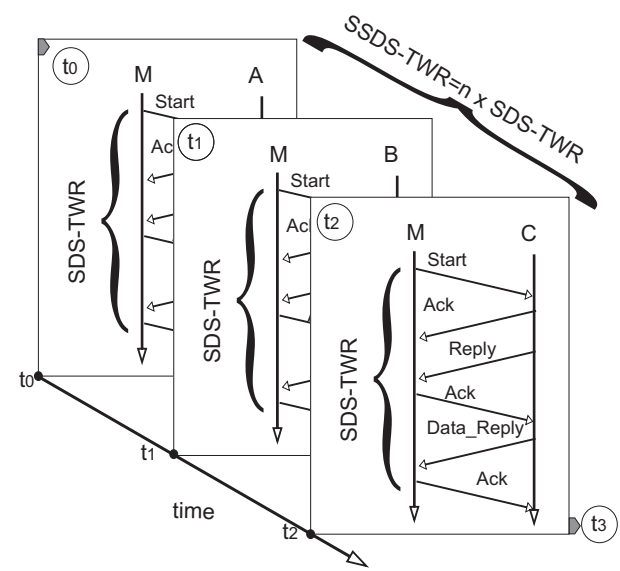

Fig. 2. Execution of Sequential SDS-TWR

\section{THE CHALLENGE}

In [14], we studied Sequential SDS-TWR (SSDS-TWR) using the Chirp Spread Spectrum (CSS) technology [1]. The idea is to execute SDS-TWR once between the mobile and each participating anchor. Figure 2 illustrates the execution of SSDS-TWR with anchors A, B and C. We proposed a new ranging protocol named Parallel DS-TWR (PDS-TWR) in order to reduce the overhead associated with localisation by replacing $2 n$ messages from the mobile to $n$ anchors with two broadcasted frames. Figure 3(a) illustrates the frame exchanges taking place in PDS-TWR. The mobile broadcasts a LocationStart message containing the list of anchors with which it wishes to perform ranging. The anchors reply one at the time according to their position in the list. Once the last Reply1 has been received or a timer has expired, the mobile sends the Data-Request: upon reception of this message, the anchors send the collected timestamps to the mobile in an orderly fashion. In [9], a theoretical study of the advantages of PDS-TWR was presented: by reducing the time spent listening for incoming frames and also sending messages, PDS-TWR reduces the energy consumption of the localisation process. The protocol specification reduces both the number and size of the messages. The expected downside is related to the precision: since PDS-TWR reuses the timestamps of the Reply1 frame for both estimations of TWR, it is more sensitive to multipath.

Figure 3(b) shows the timestamps collected for a single anchor: the mobile collects $t_{0}, t_{3}$ and $t_{4}$ while the anchor stores $t_{1}, t_{2}$ and $t_{5}$. By specifying the order of the replies, we avoid using a carrier sense scheme and do not need to execute the ranging process again to accommodate anchors which were unable to get a hold of a slot. As a matter of fact, we also defined in [14] the concept of star interval: it is a time slot similar to an IEEE 802.15.4 GTS but allowing communication between a mobile and $n$ selected anchors, forming a star topology. This star interval is created on demand and takes place during the sleep period of the network. This way, we avoid interference from the other nodes.

Although the ranging capability is very useful, from a WSN point of view, bandwidth usage is also a key aspect of every additional service. The localisation service is no exception: 

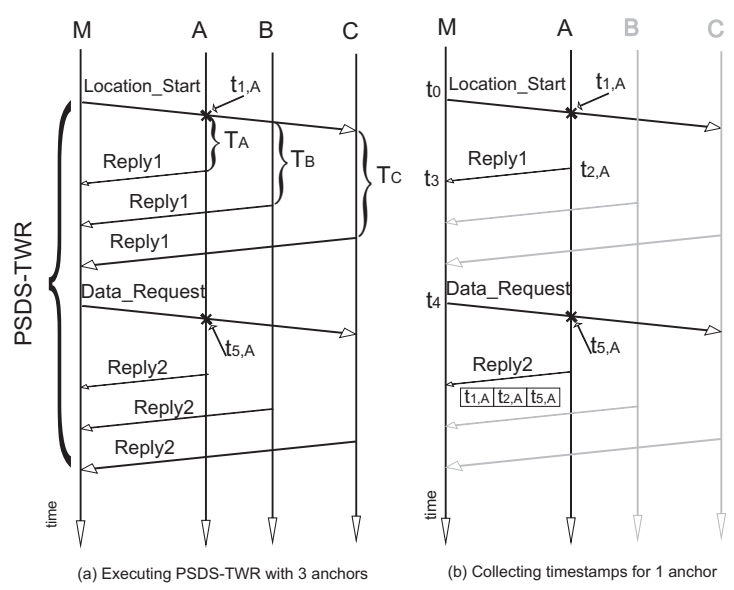

Fig. 3. Execution of Parallel Double-Sided Two-Way Ranging

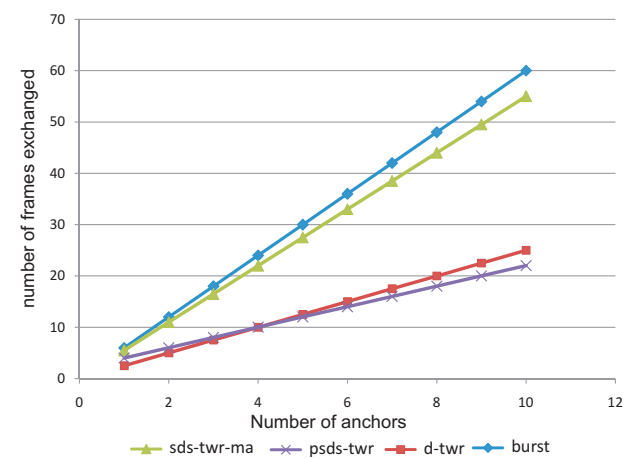

Fig. 4. Overhead comparison between SDS-TWR-MA, D-TWR, Burst and PDS-TWR

the induced overhead must be minimised. This aspect has been taken into account when designing PDS-TWR. Figure 4 illustrates the relationship between the overhead associated with PDS-TWR, SDS-TWR-MA, Burst and D-TWR. It becomes clear that the minimal overhead is obtained with PDSTWR. This reduction in medium access will lead to a cut in the consumed energy and therefore augment the lifetime of the network. Our concern is the impact of this change in the protocol on the ranging results. In this article, we will introduce the data collected from our UWB-based testbed and, through this data, study the relationship between the protocol and the ranging performance.

\section{MEASUREMEnTS AND RESUlts}

Our experiments were conducted using UWB-based nodes from [12]. These nodes operate in the $5 \mathrm{GHz}$ band with a bandwidth of $500 \mathrm{MHz}$. In our study, the microcontroller is used to control the transceiver instead of a remote computer through a Cheetah [13]. We used the following configuration in our ranging experiments: the two anchors are placed side by side and the mobile moves away from both along the line that can be drawn between the two. At predefined positions, ranging is performed. As triangle $A_{1} A_{2} P_{k}$ becomes more acute, the sides can be considered as equal to the height. In our setting (figure 5), the closest position of the mobile is $\mathrm{P} 0$ and the height is then $30 \mathrm{~cm}$. The base of the triangle, $A_{1} A_{2}$, is

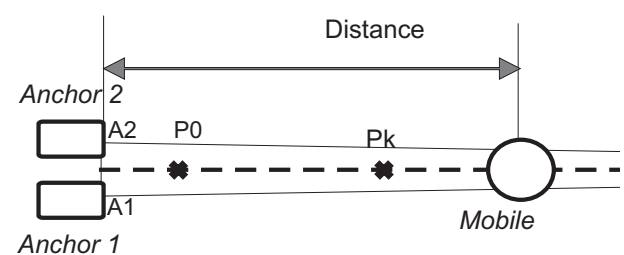

Fig. 5. Measurement settings

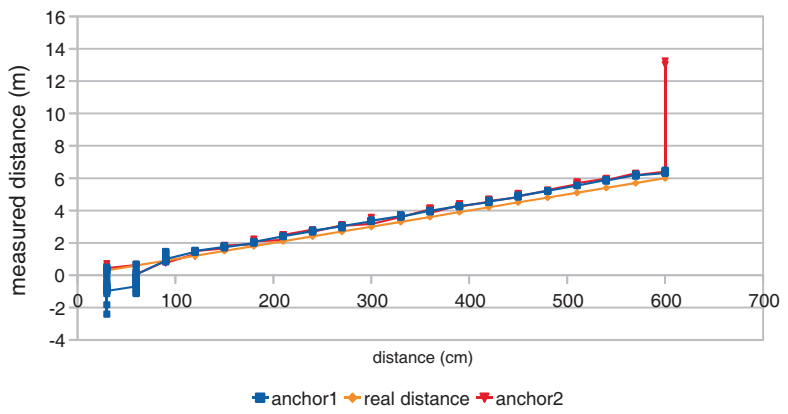

Fig. 6. Range measurements using SSDS-TWR and two anchors in an open space

$5 \mathrm{~cm}$ wide therefore the real distance from antenna to antenna is $30.1 \mathrm{~cm}$. The height $\mathrm{h}$ can be written as equation 1 :

$$
h=\sqrt{s^{2}-p^{2}}
$$

where $s$ is a side of the triangle and the real distance between the mobile and an anchor and $\mathrm{p}$ is one half of the distance between the two anchors. Since $p$ is constant, when si.ip, $\mathrm{h}$ converges towards $\mathrm{s}$. The inherent placement error will therefore be under a millimetre.

We will use the following definitions in our work: accuracy will refer to the distance between the samples and the value to be estimated. Precision will describe the scattering of the samples.

\section{A. SSDS-TWR in open space}

For the first experiment, the mobile executed SDS-TWR sequentially with two anchors, A1 and A2. As shown on figure 6 , using this protocol, the results are the same regardless of the anchor node. The mean error over this interval is $38.5 \mathrm{~cm}$ with a standard deviation of $68 \mathrm{~cm}$ for node A1. The mean error for node A2 is $45.6 \mathrm{~cm}$ and the standard deviation is $33 \mathrm{~cm}$. It is interesting to note that the slope of the resulting line is close to the slope of the real distance. This suggests that rotating the results' curve could significantly reduce the error.

We also observed that from time to time, the devices may generate an estimate which is very far from the expected values, even though SDS-TWR is used. Nevertheless, this occurs usually once for thirty samples so calculating a mean of two consecutive measurements would solve this issue.

Based on these results, we can see that SDS-TWR delivers a correct estimated distance in an indoor environment. 


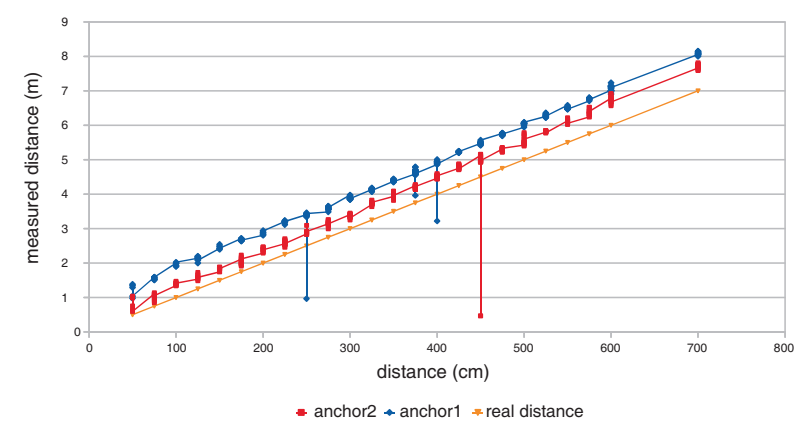

Fig. 7. Range measurements using PDS-TWR and two anchors in an open space

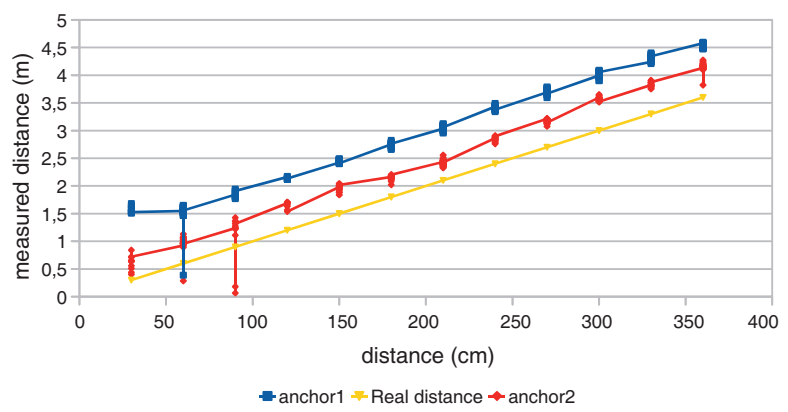

Fig. 8. Range measurements using PDS-TWR and two anchors in an anechoic chamber

\section{B. Study of the impact of PDS-TWR}

We will now investigate the behaviour of our ranging tool when using PDS-TWR. The experiment will take place in two environments. The first one is the same open space which was used for the study of SDS-TWR. The second environment is an anechoic room. This is a specially assembled room where the walls and ceiling are covered with radiation absorbent material. This product absorbs radio signals and thus shields the devices from external radiations while also eliminating multipath.

1) PDS-TWR in open space: Figure 7 shows the result of PDS-TWR using two anchors in an indoor office environment. This time, the two curves are separated by a mean distance of $45 \mathrm{~cm}$, which results in different mean ranging errors for the two anchors. The mean error associated with A1 is $94.7 \mathrm{~cm}$ while the mean error for A2 is $49.6 \mathrm{~cm}$. In this configuration, A1 is the first node to reply to the mobile. UWB being known to be robust to multipath, this difference in the behaviour should not be related to the environment but to the use of our protocol. The following section analyses the effect of a drastic environment modification on the results.

2) PDS-TWR in anechoic chamber: In order to verify the immunity of UWB to multipath, we conducted the same experiment in our anechoic chamber. The results are shown on figure 8. A similar behaviour can be observed for the two anchors. The mean ranging error for A1 is $97 \mathrm{~cm}$ and $42 \mathrm{~cm}$ for $\mathrm{A} 2$.

3) Impact of a change in the reply order: When evaluating the expected precision of a TOF-based ranging protocol, one key factor is the clock difference between the mobile and the anchor. Since our protocol introduced an order in the replies,

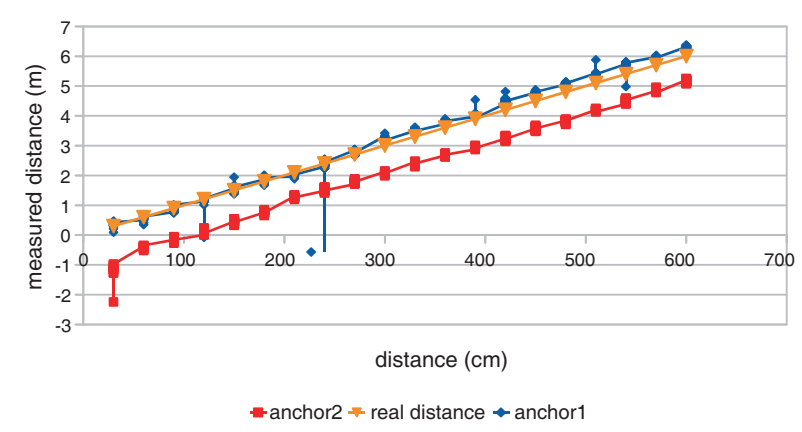

Fig. 9. Range measurements using PDS-TWR with reversed reply order

TABLE I. COMPARISON OF RANGING PRECISION OF SDS-TWR AND PDS-TWR

\begin{tabular}{|c||c|c}
\hline Scenario & Mean error(cm) A1/A2 & Standard deviation $(\mathrm{cm}) \mathrm{A} 1 / \mathrm{A} 2$ \\
\hline SDS-TWR & $0.33 / 0.38$ & $0.45 / 0.67$ \\
\hline PDS-TWR open-space & $94 / 49$ & $11 / 25$ \\
\hline PDS-TWR anechoic & $97 / 42$ & $11 / 15$ \\
\hline PDS-TWR o-s reversed & $16 /-94$ & $22 / 11$ \\
\hline
\end{tabular}

we will investigate the impact of this order on the ranging error. A2 was configured to reply before A1. The experiment was conducted in the previous open space. The results are shown on figure 9. Although the slope is still the same, the relative positions of the curves have changed. This suggests that the order of the replies also influences the nodes.

Table I summarizes the results of the experiments. The use of PDS-TWR introduces a significant ranging error, compared to SDS-TWR. We consider that both the order of the replies and clock characteristics influence the results. The distance estimates must therefore be adjusted before being submitted to the localisation algorithm. In the following section, we will study a calibration-based correction method.

\section{Proposed correction scheme}

At first, we planned on using a simple subtraction to remove the error since the slope obtained through SSDSTWR was mostly the same over the measurement interval. We then came to realise that not only did PDS-TWR have an impact on the range measurements, but the order in which the anchors were set to reply affected the results. Based on this, we decided to study a simple correction scheme. We defined a calibration position $\mathrm{P}$ in our topology. At initialisation time, the mobile is placed on this spot and it performs PDS-TWR $k$ times. Based on these measurements and the knowledge of the distance between the calibration point and the anchors, the mobile determines the error and uses the appropriate value to correct the future distance estimates.

In this experiment, we set $k$ to 6 . The topology is illustrated on figure 10 . The number of nodes being limited, the measurements were performed using only 2 anchors. Our mobile node generates a list of range measurements for each point and indicates the correction value used. To evaluate the gain, we produced a copy of the data obtained from the mobile and removed the correction. Both files were processed using the same algorithm. The results in terms of localisation error are shown on figure 11 . The error reduction varies from $41 \%$ to $60 \%$ depending on the width of the rings, which is a parameter 


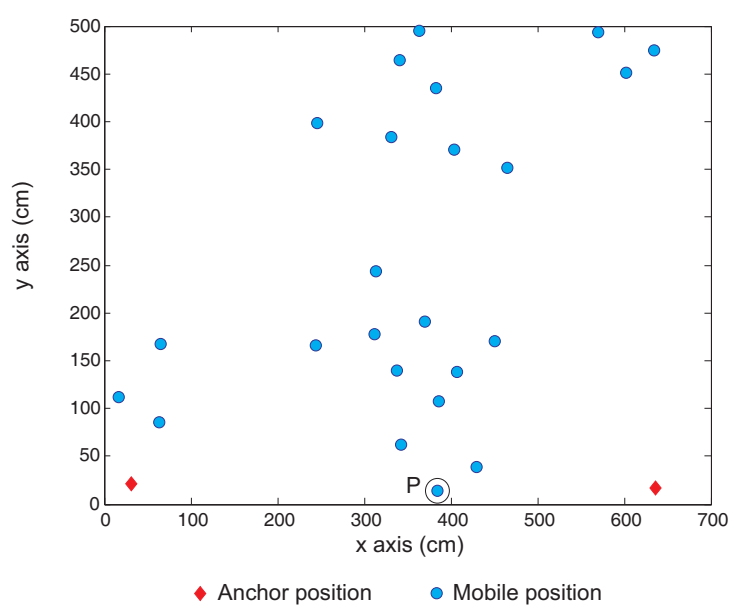

Fig. 10. Test topology

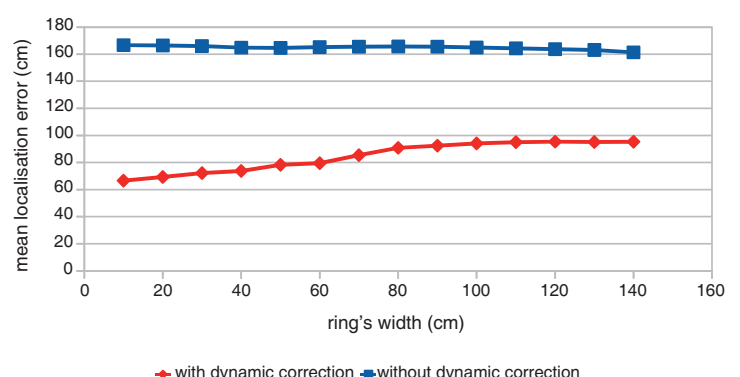

Fig. 11. Influence of the dynamic correction on localisation error

of iRingLA. Another interesting result is the reduction of cases where the position could not be computed. We often encountered the case represented on figure 12 when using the raw measurements. A ring is centred on an anchor and represents the area in which the mobile excepts to be located, based on the ranging results. The overlapping region of two or more rings corresponds to the search area of our algorithm. In this case illustrated on figure 12, an intersection cannot be found between the rings. When applying the correction scheme, the rings are shrunk using different correction factors which leads to the creation of an intersection. This reduction mostly happens for thin rings which are between $10 \mathrm{~cm}$ and $30 \mathrm{~cm}$. Out of our 6048 samples and using only two anchors, our solution can compute the position $99.9 \%$ of the time with a mean location error under $1 \mathrm{~m}$ (less than $1 \mathrm{~m}$ in $74 \%$ of the cases) in a real indoor environment. This situation corresponds to the case where a mobile reaches the border of the network coverage. Even when only two anchors are available, by using the negative information (for instance, progressive loss of radio links to other anchors), the mobile can still compute its position.

\section{CONCLUSION}

Designing an efficient localisation solution for WSNs is a real challenge today as appropriate physical layers are becoming available. The challenge is not only related to localisation performance but also to the integration of the solution in the network. We studied in this article the effect of using a ranging

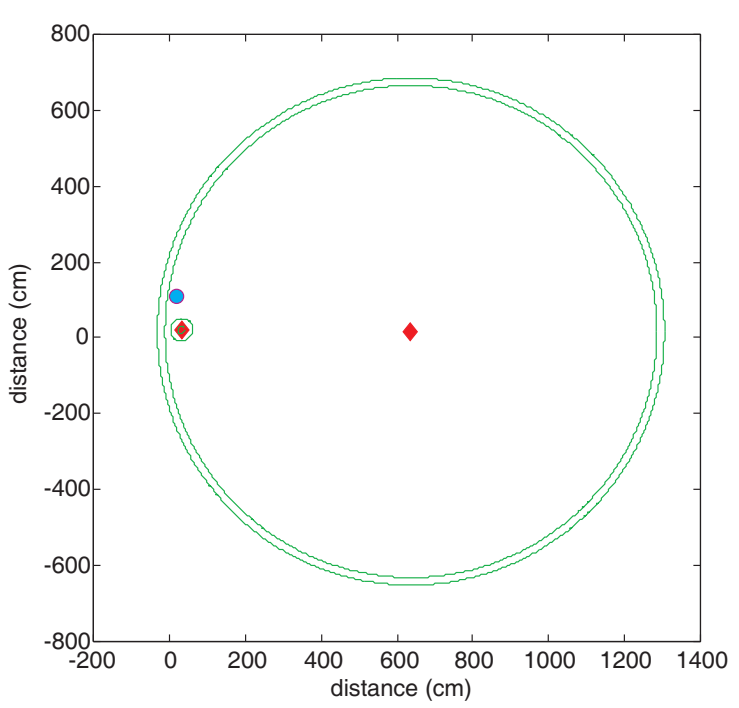

Fig. 12. Typical case which is solved using the correction

protocol which is very interesting from a network point of view on range estimation. PDS-TWR interweaves the ranging processes between a mobile and $n$ anchors. We noticed that it affects the accuracy of the estimation and proposed a correction scheme which reduces the localisation error. A more efficient correction method is currently under study and is based on the modelling of the ranging error in PDS-TWR, taking both the order of the anchors' replies and the clock characteristics into consideration. Finally, we will investigate the effect of the loss of symmetry in PDS-TWR, compared to SDS-TWR.

Nevertheless, our contribution allows easy deployment of a localisation service as it is designed to fit in the IEEE 802.15.4 stack. Overhead could be further reduced through piggybacking which is taking advantage of existing frames such as beacons or routing protocol's Hello-messages to perform ranging. This will enable innovative applications in both WSNs and the IoT. The next step is the study of the scalability of this correction scheme. In a real deployment, frequent calibration might not be feasible. Therefore, a detailed study of the source of the error in detail is necessary in order to propose a decentralised correction scheme where the anchors will compute their contribution to the error and transmit it to the mobile node.

\section{ACKNOWLEDGMENT}

The authors would like to thank Decawave for the UWB nodes and their support, and IUT de Blagnac, France, for the access to the anechoic chamber.

\section{REFERENCES}

[1] IEEE Computer Society, Specific requirements Part 15.4: Wireless Medium Access Control (MAC) and Physical Layer (PHY) Specifications for Low-Rate Wireless Personal Area Networks (WPANs) Amendment 1: Add Alternate PHYs, IEEE Standard for Information technology Telecommunications and information exchange between systems (2007)

[2] Bahl P., Padmanabhan V. N., RADAR : An In-Building RF-Based User Location and Tracking System , Infocom, mars 2000 
[3] Lanzisera S., Lin D. T., Pister K. S., RF Time of Flight Ranging for Wireless Sensor Network Localization, Workshop on Intelligent Solutions in Embedded Systems (WISES), 2006

[4] C. Liu, K. Wu, T. He, Sensor Localization with Ring Overlapping Based on Comparison of RSSI, Mobile Ad-Hoc and Sensor Systems, 2004

[5] Weidong Wang, Qingxin Zhu, High Accuracy Geometric Localization Scheme for Wireless Sensor Networks, Communication, Circuits and Systems Proceedings, 2006

[6] M. Kwak, J. Chong ,A new Double Two-Way Ranging algorithm for ranging system, 2nd IEEE International Conference on Network Infrastructure and Digital Content (2010)

[7] A.I. Baba, M.M. Atia, Burst mode symmetric double sided two way ranging, IFIP Wireless Days (WD) (2011)

[8] H. Kim, Double-Sided Two-Way Ranging Algorithm to Reduce Ranging Time, IEEE Communication Letters, Vol. 13, no. 7, July 2009

[9] R. Dalce, Localization methods using the communication signal in indoor wireless sensor networks. Phd thesis, INSA Toulouse - EDSYS Laboratories LATTIS and IRIT (2013)

[10] M. Maman, B. Denis, L. Ouvry, An Intuitive Prioritised Medium Access Scheme for Tracking Applications in UWB LDR-LT Networks, Personal, Indoor and Mobile Radio Communications (2008)

[11] M. Maman, B. Denis, M. Pezzin, B. Piagetn L. Ouvry, Synergetic MAC and Higher Layers Functionalities for UWB LDR-T Wireless NEtworks, International Conference on UWB (2008)

[12] http://www.decawave.com/about

[13] http://www.totalphase.com/products/cheetah-spi/

[14] R. Dalce, A. van den Bossche, T. Val, Indoor Self-Localization in a WSN, based on Time Of Flight: Propositions and Demonstrator, International Conference on Indoor Positionning and Indoor Navigation (2013) 